\title{
13th Aphasiology Symposium of Australia \\ October 2 and 3, 2008 The University of Queensland
}

\section{Transaction and Interaction in Severe Aphasia: Complementary or Incompatible?}

Anna Correll, ${ }^{1,2}$ Willem van Steenbrugge, ${ }^{2}$ and Ingrid Scholten ${ }^{2}$

${ }^{1}$ Repatriation General Hospital, Adelaide, Australia

2 Flinders University, Australia

\begin{abstract}
Rackground: Communication serves two main goals: transaction or infor$\boldsymbol{D}_{\text {mation exchange; and interaction or establishing and maintaining social }}$ relationships. In conversations involving individuals with severe aphasia, the strategies needed to enable successful transaction may compromise interactional goals. This study explored the relationship between interaction, transactional success, and transactional behaviours in conversations between individuals with severe aphasia and their communication partners. Method: 6 individuals with recent-onset severe aphasia and their communication partners were videorecorded in 39 conversations. Three had global and three had Broca's aphasia. Measures of transactional success and of the person with aphasia's (PWA's) and partner's interaction and transactional behaviours were obtained. The relationships between interaction and transaction measures were analysed separately for global and Broca's aphasia. Results: There was a strong positive relationship between both PWA transactional behaviours and interaction, and partner transactional behaviours and interaction, in both global and Broca's aphasia. There was no relationship between PWA transactional behaviours and partner interaction or partner transactional behaviours and PWA interaction in global aphasia, but a strong positive relationship in Broca's aphasia. There was no relationship between dyadic transactional success and either PWA or partner interaction in global aphasia, but moderate positive relationships in Broca's aphasia. Discussion: The positive relationship between own transaction and interaction suggests that adopting an approach which embraces use of skills and strategies to enhance communication in new and challenging circumstances has positive effects on both aspects of communication. In severe aphasia, use of skills and strategies by either the PWA or their conversation partner to achieve transactional success does not compromise interaction.
\end{abstract}

\section{Goals and Goal-Setting: What are the Experiences of People with Aphasia?}

Sue Sherratt, ${ }^{1,2}$ Deborah Hersh, ${ }^{1}$ Tami Howe, ${ }^{1}$ Linda Worrall, ${ }^{1}$ Bronwyn Davidson, ${ }^{1}$ and Alison Ferguson ${ }^{2}$

${ }^{1}$ University of Queensland, Australia

2 University of Newcastle, Australia

$\boldsymbol{B}_{\text {of treatment across all levels of healthcare. Goal-setting by the client is }}^{\text {ackground }}$ 
an integral part of this process but for most clients this is a complex process and often in direct contrast to their previous medical experiences. In addition, people with aphasia are impaired in the very medium necessary for negotiating and expressing their treatment goals. Methodology: Extensive qualitative data on goals and goal-setting were obtained from 50 people with aphasia using semistructured, in-depth interviewing and supported conversation techniques. These data are part of a larger, multi-site, NHMRCfunded project which includes the goal-setting experiences of the families of people with aphasia and their speech pathologists. Results: People with aphasia expressed a wide variety of rehabilitation goals post-stroke including: return to pre-stroke self, communication, information about aphasia/rehabilitation/services, independence, social and leisure activities, dignity/respect and contributing to other people with aphasia and to society. Their goals were also found to change over time as they adapted to living with chronic aphasia. The actual goal-setting process was experienced in differing ways: for some there was the extreme satisfaction of being consulted whereas others were not given the opportunity or else were unable to express their needs. Limited knowledge of their impairment, prognosis and the healthcare system contributed to the difficulties many people with aphasia experienced with goal-setting. Discussion: These findings emphasise the significance of negotiating, setting and revising rehabilitation goals for people with aphasia. Incorporating these findings into the therapy process will make rehabilitation more satisfying for all parties concerned.

\section{Goals and Goal-Setting: What are the Experiences of Family Members of People With Aphasia?}

Tami Howe, ${ }^{1}$ Bronwyn Davidson, ${ }^{1}$ Linda Worrall, ${ }^{1}$ Deborah Hersh, ${ }^{1}$ Sue Sherratt, ${ }^{1}$ and Alison Ferguson ${ }^{2}$

${ }^{1}$ University of Queensland, Australia

2 University of Newcastle, Australia

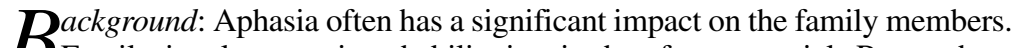
$\boldsymbol{B}_{\text {Family involvement in rehabilitation is therefore essential. Research to }}$ date, however, has not focused on the experience of goal-setting for these family members. Methodology: Semistructured in-depth interviews were conducted with 48 family members of people with aphasia in three centres in Australia. Data were analysed using Interpretative Phenomenological Analysis (Smith \& Osborn, 2003). The findings presented in this paper are part of a larger NHMRC-funded project which includes the goal-setting experiences of people with aphasia and their speech pathologists. Results: The interviews revealed several themes including the importance of information, support, and hope for family members of people with aphasia. Many participants reported that they were not always included in the rehabilitation process and that their needs were often not met. Discussion: These findings highlight the importance of speech pathologists including family members in the aphasia rehabilitation process and ensuring that their needs in areas such as information and support are addressed. 


\title{
Goals in Aphasia Therapy: What are They, How Do We Set Them and How Do We Score?
}

\author{
Deborah Hersh,, Tami Howe, ${ }^{1}$ Sue Sherratt, ${ }^{2}$ Linda Worrall, ${ }^{1}$ Bronwyn Davidson, ${ }^{1}$ \\ and Alison Ferguson ${ }^{2}$ \\ 1 University of Queensland, Australia \\ 2 University of Newcastle, Australia
}

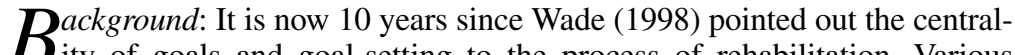
$\boldsymbol{B}_{\text {ity of goals and goal-setting to the process of rehabilitation. Various }}$ papers have focused in some detail on the goal-setting process from a range of rehabilitation perspectives. However, we still know little about how speech pathologists view goals and goal-setting in aphasia rehabilitation and the realities of how it is done in the clinic. Methodology: The data for this presentation was collected as part of a larger NHMRC-funded, multi-site project in which people with aphasia, family members and speech pathologists were interviewed about their experiences of person-centred goal-setting in aphasia rehabilitation. This presentation reflects on 40 speech pathologists' interview data, analysed using interpretative phenomenological analysis, specifically with reference to their definitions of goals and their descriptions of the goalsetting process. Results: The interviews revealed a considerable range of opinion about, for example, how goals were defined, when and how the process of goal-setting might occur, how formal it was, how it was best documented, how the process fitted with the needs of the work setting, how goals were reviewed over time and how experience changed therapists' ways of working. Goal-setting was closely related to issues of outcomes measurement, functional therapy, person-centredness, client involvement and the therapeutic relationship. Discussion: Interviewees reported positively about being given the time to consider, reflect on, and discuss such a fundamental and complex aspect of aphasia rehabilitation. It is essential for us to share ideas about real practice, what works well and how we can best incorporate quality, person-centred approaches into our rehabilitation.
\end{abstract}

\section{Goals, Metaphorically Speaking}

\author{
Alisn Ferguson, ${ }^{1}$ Linda Worrall, ${ }^{2}$ Bronwyn Davidson, ${ }^{2}$ Deborah Hersh, ${ }^{2}$ Tami Howe, ${ }^{2}$ \\ and Sue Sherratt ${ }^{2}$ \\ ${ }^{1}$ University of Newcastle, Australia \\ 2 University of Queensland, Australia
}

Background: This paper presents the preliminary results of work in
progress toward an analysis of the way people use the linguistic resource
of metaphor to describe their experience of therapy. Methodology: The
approach of critical discourse analysis is used in this research to compare the
perspectives of 3 individuals with aphasia, their spouses (3) and their
speech-language pathologists (5) about the therapy process in general and
about the therapy goals in particular. (The data is drawn from a larger
research project by the researchers listed above.) The participants were inter-
viewed by experienced speech-language pathologists with the interviews
taking about an hour. The 11 interviews were subsequently transcribed and
imported into NVivo for qualitative coding. Results: Qualitative results sug-
gest that participants overall showed some commonly shared constructs in
their perspectives on therapy, with the metaphorical concepts of 'Battle' and
'Journey' being shared across participant types. It was observed that the
people with aphasia in this study (who had mild aphasia) were able to use 
metaphoric expressions, however only participants without aphasia (family members and speech pathologists) were observed to use highly metaphoric, novel metaphors. In a single case comparison within one set of participants, it appeared they viewed therapy in very different ways. For example, the person with aphasia used Battle metaphors, while his wife's use of 'Product' metaphors showed how she felt disempowered in the system. In comparison, one of their speech pathologists described the therapy process Journey metaphors (e.g., 'tracks', 'steps', and 'building'). Discussion: While the results of the analysis of data so far is preliminary, it is suggested that collaborative goal setting can be enhanced by recognising that such metaphors offer a window into how the other partners in the process are constructing their experience.

\title{
Communicative Access in an Australian Health Network: Sustainable Systems Change in Action
}

\section{Natalie Anderson and Martin McCall-White}

Barwon Health, Australia

\begin{abstract}
Current literature reflects a growing interest in issues around access and inclusion for people with aphasia (Aphasiology, 21, 2007) in all aspects of their everyday lives. Integral to our lives is the ability to participate in and make decisions about our own healthcare. There is a growing body of literature recognising the essential role communication plays in healthcare delivery supported by legislation that makes it unlawful to discriminate against people with disabilities. Despite this, it is our experience that barriers to communicative access in healthcare still exist for a significant group of people including those with aphasia. Simmons-Mackie et al. (2007) conclude that targeting systems-level change is a useful approach to improving access to healthcare information and decision-making for people with aphasia. This prompted us to consider how communicatively accessible Barwon Health's services were and to implement sustainable systems-level changes across Barwon Health. This paper describes the development of the Communicative Access Care Improvement Group (CACIG) - a multi-disciplinary practice improvement group comprised of clinicians, managers and consumers. The primary objectives of the group are to identify potential barriers to effective communication within $\mathrm{BH}$; to motivate for change toward a communicatively accessible and inclusive health care system; and to participate in achieving this by providing ongoing education and consultation. This paper aims to provide information about the experience of communicative access in healthcare from an Australian perspective, along with an overview of the group's progress to date. Finally we will discuss lessons learned and challenges for the future.
\end{abstract}




\title{
A Home-Based Treatment Program for Word Retrieval Impairments
}

\author{
Catherine Mason, ${ }^{1}$ Lyndsey Nickels, ${ }^{1}$ Belinda McDonald, ${ }^{1}$ Melanie Moses, ${ }^{1}$ \\ Kate Makin, ${ }^{2}$ and Christine Taylor ${ }^{2}$ \\ ${ }^{1}$ Macquarie Centre for Cognitive Science (MACCS), Macquarie University, Australia \\ ${ }^{2}$ Royal Rehabilitation Centre, Sydney (RRCS), Australia
}

\begin{abstract}
$A$ ims: Therapy for word retrieval disorders has been found to be successful when delivered by clinicians. However, given the restricted access to clinical services for the majority of clients, the aim of our study was to determine if naming therapy conducted as a home treatment program could also result in significant improvements in lexical retrieval. Methods and Procedures: Three people with aphasia participated. They each selected 60 treatment words that they wished to be able to retrieve more successfully. These words could be broadly divided into 3 topic sets for each participant. To improve word retrieval they were provided with a home treatment programme which implemented a technique (repetition) which had been found to be successful with these participants previously. Multiple baselines were recorded to observe for any change in naming accuracy without treatment. Structured conversations based around each of the three topic sets were sampled prior to treatment and again following treatment to see if there was any generalization to conversation-level communication. Results: The participants showed increased naming accuracy following the home program. While there was no significant increase in production of the treatment words in conversation, other changes in conversation-level communication were apparent including improved turn taking and increased use of content words. Discussion and Conclusions: This study shows that there is potential for naming therapy to be conducted as a home programme. However, the home treatment program overall showed less robust changes than the previous clinician-administered program. Two possible reasons for this are adherence to the treatment protocol and the nature of the stimuli treated in each program.
\end{abstract}

\section{Interventions in Semantic Dementia and Progressive Aphasia: Clinical and Theoretical Issues}

\author{
Karen Croot ${ }^{1}$ and Lyndsey Nickels ${ }^{2}$ \\ ${ }^{1}$ Department of Psychology, University of Sydney, Australia \\ ${ }^{2}$ Macquarie Centre for Cognitive Science (MACCS), Macquarie University, Australia.
}

espite the core language symptoms in primary progressive aphasia, semantic dementia and nonfluent progressive aphasia, people with these disorders are likely to be under-referred for speech pathology services, and there is limited published research on appropriate interventions in these disorders. This paper reviews the published impairment- and activity/ participation-based interventions carried out with people with these disorders, to determine the current evidence base for clinical decisions about client suitability and selection of treatment goals, methods and measures. We also identify questions that need to be addressed in future therapy research. Two-thirds of the impairment-based interventions targeted object/picture naming, but knowledge of semantic attributes, adjective and verb retrieval, use of tense markers, auditory-phonological processing and dysgraphia have also been treated. The observed patterns of therapy gain, limited generalisation, and decline following cessation of therapy raise questions about the learning mechanism(s) involved, and have implications for client suitability and choice of therapy items, therapy delivery and dura- 
tion. There are few systematic activity/participation-based interventions, although the available reports suggest increased communicativeness and communication effectiveness for participants following intervention, especially on everyday communication outside the clinic, but these impacts need to be documented. These studies highlight the need for rigorous research design to identify treatment, generalisation and maintenance effects, as well as outcomes that go beyond improvement in the clinic on targeted structures/behaviours. It is important to tailor interventions closely to individual client needs, involve the spouse/carer in intervention, and ensure clients have appropriate expectations about therapy.

\title{
Functional Communication Group Therapy
}

\author{
A. Correll, C. Hopkins, M. Schar and A. Crockett-Naini \\ Repatriation General Hospital, Adelaide, Australia
}

$\boldsymbol{B}_{\text {guage therapy resources and support social interaction, as well as pro- }}$ viding an economical method of service delivery. Research exploring group therapy for patients with aphasia has highlighted improvements in functional communication, linguistic structure, and psychological wellbeing. This project developed a functional group program for in and out patients with mixed communication impairments. The therapy modelled functional communication situations and tasks needed for community living and social integration. Each group consisted of a maximum of 5 patients, with 3 concurrent groups (talking, reading, writing) rotating through a 6-week cycle. The program content catered for varying levels of communication impairment severity, with each therapy task also having corresponding levels of difficulty. Methodology: 57 patients with communication impairments, including aphasia, cognitive-communication disorders, dysarthria, and apraxia of speech, and with severity ranging from mild to severe, participated in this project. The communication confidence profile (CCP) was used pre and post group intervention to measure change in patients' confidence when communicating. Patient satisfaction with the groups was also measured. Results: The results indicated improvement across all 3 modalities (talking, reading and writing). Patients indicated the greatest change with talking, followed by reading and writing respectively. Patients were, overall, satisfied with the group process. Discussion: The potential benefits of a group therapy approach include enhanced social confidence and social integration, which assists community living. This program increased therapy options for patients, and provided a cost effective service. It incorporated functional tasks, and produced improvements in communication confidence for patients with mixed communication impairments. 


\title{
Assessing Aphasia in Arabic Speakers: Work in Progress
}

\author{
Samar Al-Amawi, Alison Ferguson and Sally Hewat \\ University of Newcastle, Australia
}

$\mathrm{T}$

This research aims to find out the most effective assessment procedure to assess Arabic speaking individuals with aphasia who live in a country with a different linguistic and cultural background. The research will focus on this process within the ICF (ICIDH2) framework, with reference to the Arabic culture background and beliefs regarding illness, disablement, the available health services, and the consequences of the individual's language disablement on his/her everyday life activities and quality of life. Ethnographic research seems to be the most appropriate method that may allow for the description of the Arabic aphasic individual's functional communication, through describing everyday activities and needs, and the communicative contexts that he/she may participate during everyday life within the seven variables: place, time, communication, partner, topic, duration, interactional/ transactional components, and mode of communication. As has been stated by Worrall (1999), we have to involve the person with aphasia in describing the activities that are relevant to his/her everyday life, and this research will attempt to do this through an interpreter-mediated interview. Kagan and Gailey (1993) stated that pragmatic assessments and conversational or discourse analyses are more 'functional' than contextual tests, and with this in mind some crucial questions arise when we think about assessing Arabic aphasic speaker: How can we establish or create a functional assessment tool that can be used with Arabic individuals with aphasia? How can the interpreter describe or analyse the Arabic speaker's conversational speech when the speech pathologist's first language is not Arabic? This paper will discuss these questions and the plans for future research in this area.

\section{How Does Our Impairment-Focused Therapy Work?}

Lyndsey Nickels

Macquarie Centre for Cognitive Science (MACCS), Macquarie University, Australia

Tt is now well established that we can improve word retrieval as a result of Iseveral different treatment techniques. Here, we focus not on whether or not we can effect improvements in word retrieval, but the mechanisms that might underlie such changes.It has long been known that having once produced a word, this word will be produced more easily on a subsequent occasion. The mechanisms that underly this phenomenon are referred to as priming mechanisms. More recently, these priming mechanisms have been also suggested to account for improvements in word retrieval in people with aphasia. This paper will also describe some recent experiments with unimpaired speakers, which demonstrate that priming can, on occasions, impair naming as well as improve it. The implications of the findings for treatment of word retrieval impairments in aphasia will be discussed. 


\title{
A New Tool to Examine Single Case Experimental Designs: The SCED Scale
}

Leanne Togher, ${ }^{1}$ Robyn Tate, ${ }^{2,3}$ Michael Perdices, ${ }^{4}$ Skye McDonald, ${ }^{5}$ Regina Schultz ${ }^{2,3}$ and Sharon Savage $\mathrm{e}^{2,3}$

\author{
1 Speech Pathology, Faculty of Health Sciences, The University of Sydney, Australia \\ ${ }^{2}$ Rehabilitation Studies Unit, Northern Clinical School, Faculty of Medicine, University of Sydney, Australia \\ ${ }^{3}$ Royal Rehabilitation Centre, Sydney \\ ${ }^{4}$ Department of Neurology, Royal North Shore Hospital, Sydney, Australia \\ ${ }^{5}$ School of Psychology, University of New South Wales, Australia
}

\begin{abstract}
$\mathrm{W}$ ith the advent of evidence based practice it is now an expectation that clinicians and researchers evaluate the methodological quality of treatment studies as part of the clinical decision making process. Over the last decade various systems have been developed to determine the quality of evidence, including guidelines from the NHMRC (2000), the Academy of Neurology (2004) and the CONSORT statement (Moher, Shulz \& Altman, 2001). In Australia, two teams of clinical researchers have developed additional tools to evaluate methodological quality of research; the PEDro team (www.pedro.fhs.usyd.edu.au) has developed the PEDro scale to evaluate group designs and the PsycBITE ${ }^{\mathrm{TM}}$ team (www.psycbite.com) has developed the Single-Case Experimental Design (SCED) scale to evaluate single case experimental design studies. Both these scales will be used in evaluating papers on the speechBITETM database (www.speechbite.com). The methodological quality of aphasia group design studies has been assessed using the PEDro scale (Togher et al., in press). Of 339 studies indexed for aphasia 70\% were single case experimental designs (SCEDs). Given the significant proportion of SCEDs, the PsycBITETM team has developed a scale which examines the methodological quality of single case experimental designs (Tate et al., 2008). This paper will present the items on the SCED scale and the results of 2 reliability studies which attest to the psychometric robustness of this scale. Tools which evaluate methodological quality are useful for a number of reasons. They provide clinicians with a way of determining the quality of papers as part of their clinical decision making. They give researchers benchmarks against which to develop well designed clinical research studies and provide a common language for clinicians and researchers to discuss the relative merits of published research. Finally, it is hoped their use will ultimately improve the robustness of treatment outcome studies and therefore improve quality of life for people with aphasia.
\end{abstract}

\section{Hemispheric Contributions to the Recovery of Comprehension in Aphasia: A Divided Visual Field Investigation}

Erin Smith, Helen Chenery, Anthony Angwin and David Copland

The University of Queensland, Australia

$\mathrm{H}$ emispheric contributions to semantic processing were investigated for people with chronic aphasia using a divided visual field (DVF) priming paradigm. Previous investigations of healthy individuals suggest that both the left hemisphere $(\mathrm{LH})$ and right hemisphere $(\mathrm{RH})$ play a role in semantic processing. The concept that the RH could compensate for language impairment following LH stroke, is the motivation for this investigation. The current investigation aimed to explore the impact of $\mathrm{LH}$ lesions on the processing of category members during a semantic priming DVF task. Controlled semantic priming was targeted via a high relatedness proportion, and time-course explored using two stimulus onset asynchronies (SOA; 
$250 \mathrm{~ms}$ and $750 \mathrm{~ms})$. The control group revealed bilateral priming for both the category associated (CA) and category nonassociated (CO) conditions, while the $\mathrm{LH}$ lesion group demonstrated bilateral activation in the CA condition at both SOAs. In order to explore the impact of hemispheric contributions to the success of recovery, the LH lesion group were split into high and low comprehension groups. The high comprehension group revealed priming exclusive to the LVF/RH, for both $\mathrm{CA}$ and $\mathrm{CO}$ conditions at the short SOA, and RH CA priming at the long SOA. The low comprehension group showed priming for CA RVF/LH targets only, at both the long and short SOA. These findings indicate the importance of the RH for successful comprehension recovery following LH lesions, as well as highlighting the importance of the associative relationship to encourage semantic activation in people with a LH lesion.

\title{
Plural Errors in Aphasic Speech: How is 'Number' Represented in the Mental Lexicon?
}

\author{
Britta Biedermann, Lyndsey Nickels and Anna Elisabeth Beyersmann \\ Macquarie Centre for Cognitive Science (MACCS), Macquarie University, Australia
}

$\boldsymbol{B}_{\text {ackground: This study uses plural errors of aphasic speakers to explore }}$ are two major cognitive plural production theories. The decompositional account assumes a rule-based process for the plural marking (stem + -s; e.g., Schreuder \& Baayen, 1995), whereas irregular plural forms have to be fully listed (e.g., Butterworth, 1983). This study applies these two theories to the plural marking of nouns which occur more frequently as plural form (plural-dominant nouns, e.g. 'eyes') and compares them to the plural form of nouns which are more frequent in their singular form (singular-dominant;e.g., 'noses'). Methodology: 2 aphasic speakers, FME and DRS, were required to name pictures depicting either 'single' or 'multiple' objects (e.g., picture of ' 1 cat', or picture of ' 4 cats'). Both aphasic speakers were at least one year post-onset; impaired in word production and produced number errors (e.g., singular produced as plural or vice versa). Results: DRS showed significantly more errors when asked to name multiple entities of 'singular-dominant' items, but showed the reversed pattern for the 'plural-dominant' item group: more number errors occurred when the target picture was represented as a single entity. In contrast, FME showed always more errors for the plural form, regardless of whether the noun was singular - or plural-dominant. Discussion. Levels of breakdown and theoretical implications for functional models (e.g., Levelt et al., 1999) are discussed in order to explain the influence of 'plural dominance'. 


\title{
Language Switching in Bilingual Aphasia: What is 'Pathological' Code-switching?
}

Amanda Miller Amberber, Lyndsey Nickels, Max Coltheart and Stephen Crain Macquarie Centre for Cognitive Science (MACCS), Macquarie University, Australia

\begin{abstract}
B ilingual and multilingual speakers may alternate between languages within a conversation and within a sentence. This is called code-switching. Fluent adult bilinguals who have acquired both languages in early childhood, code-switch in accordance with conversational discourse/pragmatic principles and with grammatical principles, like those that apply in single languages. The question then arises: do bilingual aphasic speakers demonstrate 'pathological' code-switching, that is, code-switching that fails to comply with these conversational and grammatical principles? Previous research has shown that some aphasic bilinguals manifest conversationally impaired code-switching. Few studies have directly addressed grammatical impairment of code-switching, and these studies have inadvertently applied invalid measures of what constitutes grammatical code-switching in healthy proficient bilingual speakers. Here we report the results of a study investigating the grammatical and conversational patterns of code-switching for 2 aphasic bilingual speakers, in comparison with language- and age-matched controls. Code-switching was investigated across five measures (conversation, narrative recount, elicited sentence production, lexical selection and grammaticality judgements) for three sentence types (subject pronoun declaratives, negation, wh- questions). Testing of lexical, syntactic and cognitive abilities was completed in each language. The results demonstrated a clear distinction between conversational impairment and grammatical impairment of code-switching. For these bilingual aphasic speakers, grammatical impairment of code-switching was evidenced in the absence of conversational impairment. Clinical and theoretical implications of the results will be discussed.
\end{abstract}

\section{Production of Syntactically Complex Verbs in Aphasia: A Comparison of Sentence and Discourse Contexts}

Wiebke Grauel, Linda Cupples and Elizabeth Armstong

Department of Linguistics, Macquarie University, Australia

Background: Production of passives has been found to be difficult for
individuals with nonfluent aphasia but has traditionally been assessed
with verbs whose canonical structure is that of an active (e.g., agent-theme
verbs, such as 'climb'). Different types of verbs (e.g., theme-experiencer
verbs, such as 'frighten') have, however, been found to be more commonly
produced in the passive voice by nonbrain-damaged (NBD) individuals. This
study investigated whether people with non-fluent aphasia would also find it
easier to produce grammatical passives with theme-experiencer verbs than
with agent-theme verbs. Methodology: Preliminary data were collected
from two individuals with aphasia: P1 with very mild nonfluent aphasia, and
P2 with moderate to severe nonfluent aphasia. A small group of six NBD
individuals was also included. Production of passives was elicited by means
of a picture description task comprising two conditions with different levels
of constraint, as well as a highly constrained sentence anagram task. Results:
The highly constrained sentence anagram task elicited grammatical passives
from all three participants with both agent-theme and theme-experiencer 
verbs. On the other hand, none of the participants produced passives with agent-theme verbs in the unconstrained condition of the picture description task. In the more constrained condition, both P1 and the NBD group produced passives with agent-theme verbs, whereas P2 continued to avoid passive structures with this verb type. By contrast with these results for agent-theme verbs, the majority of responses to theme-experiencer verbs were grammatical passives for all participants, including $\mathrm{P} 2$, in both the less constrained and the more constrained picture description conditions. Conclusion: P2's results for theme-experiencer verbs suggest that production of passives by individuals with non-fluent aphasia is largely preserved in the context of verbs whose preferred structure is that of a passive. Furthermore, an effect of task constraint was observed, not only for individuals with aphasia but also for the NBD group.

\title{
Life Storybooks and DVDs to Increase Communicative Participation Across Disease Progression in Huntington Disease: A Case Discussion
}

\author{
Emma Power, ${ }^{1}$ Alison Anderson ${ }^{2}$ and Leanne Togher ${ }^{1}$ \\ ${ }^{1}$ Speech Pathology, Faculty of Health Sciences, University of Sydney, Australia \\ ${ }^{2}$ Huntington Disease Service, Sydney West Area Health Service, NSW Health, Sydney, Australia.
}

पuntington's disease is a fatal, hereditary neurodegenerative disorder - 1 characterised by motor, cognitive and psychiatric symptoms that impact dramatically on an individual's ability to communicate. These same symptoms, along with the hereditary nature of the disease may also affect the success of techniques used to maximise a person's communicative participation. A life-story approach, based on the WHO ICF framework, was trialled with a young man with HD in a residential care setting as an alternative and augmentative communication (AAC) tool to meet his immediate and longterm communicative needs. Communication assessment consisted of qualitative interviews with the client and his mother supplemented with formal and informal assessments of cognitive-linguistic and speech motor skills. His current communicative strengths and limitations were explored, along with anticipatory discussions on communicative and overall decline in functioning in the context of relevant Environmental and Personal Factors. Production of a life story book (LSB) and DVD was jointly agreed to as the best method to maximise his communicative opportunity and capacity as well as to preserve self-identity with his eventual communicative decline. Content themes analysed from qualitative interviews with the client and his mother on completion of the LSB and DVD and as the disease progressed, indicated the approach based on the ICF framework had multiple applications and values in terms of communicative participation. The presentation will trace the use of both the WHO ICF and life-story approach in a client-clinician partnership for long-term communication planning and intervention. 


\title{
Clinical Application of 'Evidence' in Aphasia: The PAH Experience
}

Jenny Lethlean and Joanna Rhee

Speech Pathology Department, Princess Alexandra Hospital, Brisbane, Australia

\begin{abstract}
Clinical aphasiologists are more commonly implementing the World Health Organisation International Classification of Funtioning model into practice through applying research recommendations at impairment, activity and participation levels. Conferences such as the Aphasiology Symposium of Australia (ASA) provide an opportunity for clinicians to identify recommendations relevant for their clinical setting. The implementation of different models of aphasia therapy, use of aphasia friendly resources, introduction of errorless learning treatment for anomia, formalised communication partner training and the importance of aphasia support groups and the advocacy role of clinicians addressing the environmental barriers to living with aphasia are examples from the 2006 ASA conference which have been adopted by the Speech Pathology Department at the Princess Alexandra Hospital. The enablers and challenges in the implementation of changes to aphasia management in the clinical setting will be discussed. The importance of optimising the reciprocal relationship between evidence based practice and research will be highlighted in a practical way.
\end{abstract}

\section{Barriers and Facilitators to Mobile Phone Use for People With Aphasia}

Carole-Ann Greig, Renee Harper, Tanya Hirst, Tami Howe and Bronwyn Davidson The University of Queensland, Australia

ackground: Mobile phone use may be beneficial in facilitating social
participation. People with aphasia are disadvantaged in the use of infor-
mation and communication technology such as mobile phones and are
reported to be more socially isolated than their peers. The World Health
Organization's International Classification of Functioning, Disability and
Health provides a framework to address the impact of environmental factors
on individual participation. The aim of this preliminary study was to identify
the barriers and facilitators to mobile phone use for people with aphasia.
Methodology: A qualitative descriptive study involving two phases was con-
ducted: (1) semi-structured interviews with six individuals with aphasia who
owned or expressed a desire to own a mobile phone; (2) structured observa-
tions of key scenarios identified in the interviews of three participants who
were sampled from the interview study (Greig, Harper, Hirst, Howe, \&
Davidson, in press). Results; Results identified 18 barriers and 9 facilitators
to mobile phone use. Key barriers and facilitators were identified in the areas
of design and features, written support and training, and communicative
partners. Discussion: Mobile phone use can be problematic for people with
aphasia. Intervention needs to address the barriers and utilise the facilitators
to mobile phone use for this population. Further research is required to
inform policy and intervention programs to ensure that people with aphasia
have access to this technology. 


\section{Speaking Aphasian}

Claire Esgate and Julia Murphy, with the Talk Time Group

Royal Rehabilitation Centre Sydney, Australia

Rackground: The Life Participation Approach to Aphasia (LPAA) is a B.consumer-driven service delivery philosophy that focuses on maximising re-engagement in life for all those affected by aphasia'. It focuses on supporting those who are affected by aphasia to achieve their real life short and long-term goals, and recommends that clinicians consider both transaction and interaction in therapy (LPAA Project Group, 2000). In 2008, the Royal Rehabilitation Centre Sydney (Royal Rehab) speech pathology service reestablished the Talk Time outpatient aphasia group within the LPAA model. The clinicians aimed to explore the role of supporting and measuring group driven goals, and facilitating social links within and beyond the group. Methodology: Outpatient and community clients with chronic aphasia of all types and severity levels were invited to attend the group. Clients were invited to identify communication-related goals for themselves and to discuss group-related goals for each block. Results: In the first block, the group agreed to focus on the development of 'life stories' with the aim to share about themselves in the group, and reflect on their experiences of life with aphasia. This led to discussion about aphasia education and the group members' role as 'experts' in aphasia. The group agreed to spend the next block discussing and developing a resource with information and tips about aphasia - from the perspective of people with aphasia. Expected outcomes included opportunities to share experiences, develop in confidence and advocacy skills, and improve social links and community education through development of a resource on the personal experience of life with aphasia. Discussion: Using the LPAA model in an aphasia group setting led to identification of a group goal and project which would help members to share their experiences of aphasia with their family and friends in a meaningful way, and educate their community (including health professionals) on the personal experience of aphasia. 